\title{
In diffuse intrinsic pontine gliomas, non-coding RNA expression is significantly varied dependent on overall survival
}

Henley Castillo

Kenzie Mellor

Amelia-Mae Marks

Willard L ( $\sim$ rp311701@outlook.com )

Research Article

Keywords: Cancer, RNA, transcriptome

Posted Date: June 7th, 2021

DOI: https://doi.org/10.21203/rs.3.rs-592389/v1

License: (c) (1) This work is licensed under a Creative Commons Attribution 4.0 International License.

Read Full License 


\section{Abstract}

Diffuse intrinsic pontine glioma is a kind of pediatric brain cancer that kills 99 percent of patients within five years and for which there are no conventional chemotherapies. It is crucial for new treatments to comprehend the cancer's transcriptional activity. Using a published dataset, we compared the transcriptomes of tumors from patients who lived longer or less than six months. Among the genes whose expression changed most, we observed that numerous microRNAs and snoRNAs were present. The publication's findings are the first evidence of variable levels of non-coding RNA expression in diffuse intrinsic pontine glioma.

\section{Introduction}

Diffuse intrinsic pontine glioma is a kind of juvenile brain cancer that develops from glial cells in the brainstem $(1,4,5)$. DIPG patients have the worst prognosis of any cancer diagnosis: 99 percent will die within five years, and 100 percent will die within ten years (1). There is no recognized standard chemotherapy (2), even for palliative usage, and radiation therapy is the sole treatment regimen that is deemed conventional (6). Understanding the fundamental biology of cancer is essential for identifying treatment targets and developing innovative therapy regimens. To that goal, we used a published dataset to do a global, differential gene expression study of tumors from DIPG patients. We discovered that many non-coding RNA transcripts were in the top $1 \%$ of transcripts that were differently expressed between these two groups of patients with varied overall survival outcomes by comparing the transcriptomes of patients who lived longer or less than six months. MicroRNA and short nucleolar RNAs, or snoRNAs, were among them. These findings are the first to show differences in non-coding RNA expression in DIPG dependent on patient outcomes.

\section{Method}

Differential gene expression analysis was performed on tumor samples from patients diagnosed with DIPG $(n=35 ; n=21$ for patients surviving longer than 6 months, and $n=14$ for patients surviving less than 6 months) using dataset GSE50021 (3) in combination with GEO2R. In this dataset, there was one patient who lived for precisely six months and was included in the group of patients who lived for fewer than six months. Buczkowicz et al. (3) obtained transcriptome data from these cancers and control brain tissues using Illumina HT-12 microarray analysis. Fresh frozen paraffin embedded tumors from juvenile patients or normal healthy brain tissue were used in all of these samples. The p-value was not altered in any way. The log transformation was turned off, and the platform annotation category provided by the submitter was utilized. An unpaired, two-tailed t-test with Welch's correction was employed for statistical analysis to compare the RNA expression levels across patient groups (PRISM 8.1.2). (227). PRISM was also used to do linear regression analysis and a Pearson correlation, which compared each patient's mRNA expression level to the length of time they lived.

\section{Result}


We used differential gene expression analysis to find the genes whose expression differed the most between the tumor transcriptomes of patients who survived more than six months and those who didn't. Interestingly, eight distinct non-coding RNA transcripts were within the top $1 \%$ of differentially expressed transcripts. Five snoRNAs and five microRNAs were among them. Non-coding RNA, such as snoRNA and microRNA, account for ten of the top $1 \%$ of differentially expressed genes when comparing the transcriptomes of people who live longer or shorter than six months. Five distinct small nucleolar RNAs, or snoRNAs, were found to be in the top $1 \%$ of transcripts differentially expressed in the tumor transcriptomes of patients who lived longer or shorter than six months. Out of the 29285 total transcripts discovered and quantified in the microarray data studied in this research, SNORD94 was the 14th most differentially expressed gene (3). Out of 29285 total transcripts discovered and quantified, SNORD100 was the 30th most differentially expressed gene. Out of 29285 total transcripts discovered and quantified, SNORD 65 was the 65th most differentially expressed gene. Out of 29285 total transcripts discovered and quantified, SNORD49A was the 200th most differentially expressed gene. There were five microRNAs in the top $1 \%$ of transcripts differentially expressed between tumor transcriptomes from patients who survived more than 6 months and those who did not. Out of 29285 total transcripts discovered and analyzed, miR939 was the 15th most differentially expressed gene. Out of 29285 total transcripts discovered and quantified, miR429 was the 29th most differentially expressed gene (Table 1; $\mathrm{p}=0.007442$ ). Out of 29285 total transcripts discovered and quantified, miR595 was the 69th most differentially expressed gene (Table $1 ; p=0.012938$ ). Out of the 29285 total transcripts discovered and quantified in the microarray data evaluated in this research, miR154 was the 140th most differentially expressed gene. Out of 29285 total transcripts discovered and quantified, miR485 was the 197th most differentially expressed gene $(p=0.023651)$. Following that, we examined the expression levels of each non-coding RNA transcript in the tumors of all 35 patients in two groups: those who survived more than 6 months and those who did not. In the tumors of patients who survived more than 6 months, each of five microRNA transcripts was expressed at much lower levels. SNORA94 expression was found to be considerably lower in the tumors of patients who lived longer than six months. In the tumors of patients who survived more than six months, SNORD65 was expressed at much lower levels. In the tumors of patients who survived more than six months, SNORD100 was expressed at much lower levels. In the tumors of patients who lived longer than six months, SNORD49A was expressed at lower levels.

SNORA22 expression was found to be considerably higher in the tumors of patients who lived longer than six months. In the tumors of patients who survived more than six months, all but one snoRNA, SNORA22, was expressed at reduced levels. Finally, we looked to see whether the expression of any of these noncoding RNA transcripts had a linear relationship with the patient's overall survival, as defined by the amount of time they lived. SNORA22 was the only ncRNA whose expression had a linear and non-zero connection with patient survival and reached the threshold of statistical significance with a $95 \%$ confidence interval. Surprisingly, out of 10 ncRNA transcripts, this was the only one whose expression was higher in the tumors of patients who survived longer than six months.

\section{Conclusions}


In this work, we used global differential gene expression profiling to better understand the transcriptional activity of diffuse intrinsic pontine gliomas in the brainstem. We discovered that 10 of the top $1 \%$ of transcripts that were most differentially expressed between tumors of patients who survived greater or less than six months were non-coding RNA transcripts, both microRNA and snoRNAs, by comparing the transcriptomes of tumors from patients who survived greater or less than six months. These non-coding RNA transcripts may be employed as a diagnostic tool. More specifically, SNORA22 levels were significantly higher in tumors of patients who survived for more than 6 months, and SNORA22 levels in each patient's tumor also displayed a linear correlation with the amount of time each patient survived, though this correlation was not statistically significant to 95 percent confidence. SNORA22 is a member of the H/ACA box of snoRNAs, which direct the conversion of uridine to pseudouridine in substrate RNAs, primarily ribosomal RNAs (rRNAs) (7). Exosomal RNA SNORA22 has been shown to be overexpressed in pancreatic ductal adenocarcinoma (PDAC) (8). In a study of prostate cancer xenografts, SNORA22 was discovered to be expressed differently in metastatic and non-metastatic xenografts (9). Breast cancer (10), ovarian cancer (11), gastric cancer (12), and hepatocellular carcinoma (12) have all been linked to miR939 (13). Prostate cancer $(14,15)$, colorectal cancer (16), hepatocellular carcinoma (17), and nonsmall cell lung carcinoma (18) have all been linked to miR154 (18). Nonsmall cell lung cancer (19), gastric carcinoma (20), ovarian cancer (21), colorectal cancer (22-23), bladder cancer (24), breast cancer (25), and esophageal carcinoma (26) have all been linked to miR429 expression (26). Breast cancer (2729), gastric cancer $(30,31)$, bladder cancer (32), lung adenocarcinoma (33), liver cancer (34), prostate cancer (35), and hepatocellular carcinoma (36), have all been linked to miR485 in some way (36, 37). One intriguing miR485 regulatory circuit included NF-YB regulation by miR-485-3p, which ultimately resulted in variations in DNA topoisomerase II expression, resulting in variations in responsiveness to topoisomerase inhibitor (38). Both rhabdomyosarcoma Rh30 cell and the leukemia CEM/VM-1-5 cell line were used in these experiments (38). In terms of miR595, the transcription factor Sox7 was regulated by mir595, which aided the growth of human glioblastoma cell lines (39). Ovarian cancer (40), neuroblastoma (41) and hepatocellular carcinoma (42) have all been linked to miR595 expression (42). We discovered that individuals with diffuse intrinsic pontine glioma differently express a number of non-coding RNA transcripts, including microRNAs and snoRNAs, by analyzing the transcriptomes of tumors from patients who lived longer or less than six months. Except for one ncRNA transcript, SNORA22, all 10 differentially expressed ncRNA transcripts were significantly lower in the tumours of patients who survived more than 6 months. The length of time each patient lived (overall survival) was linearly associated with the expression of SNORA22 and SNORA22 alone, and this association approached but did not reach statistical significance (95 percent confidence). All of the microRNAs that were shown to be differently expressed in DIPG depending on patient outcomes have previously been linked to cancer etiology in a variety of malignancies. This is the first study to show that the expression of these microRNAs differs depending on the patient's outcome in DIPG. These findings warrant further investigation into the contribution of each of these microRNA and snoRNA in the etiology of DIPG, as well as whether any of them can be used to stratify patients at the time of diagnosis into groups who will have more severe disease courses and may benefit from more intensive radiation therapy. Understanding the disease's 
transcriptional landscape is the initial step in identifying treatment targets and developing new treatment methods for DIPG patients.

\section{Declarations}

Competing interests: We declare no competing interests

\section{References}

1. Waks AG, Winer EP. Breast cancer treatment: a review. JAMA. 2019;321:288-300.

2. Burstein MD, et al. Comprehensive genomic analysis identifies novel subtypes and targets of triplenegative breast cancer. Clin. Cancer Res. 2015;21:1688-1698.

3. Aggarwal C, et al. SWOG S1400D (NCT02c965378), a phase Illstudy of the fibroblast growth factor receptor inhibitor AZD4547 in previously treated patients with fibroblast growth factor pathwayactivated stage IV squamous cell lung cancer (lung-MAP substudy) J. Thoracc. Oncol. 2019;14:1847-1852.

4. Edelman MJ, et al. SWOG S1400C (NCT02154490)-a phase III study of paalbociclib for previously treated cell cycle gene alteration-positive patients with stage IV squamous cell lung cancer (lungMAP substudy) J. Thorac. Oncol. 2019;14:1853-1859.

5. Herbst RS, et al. Lung master protocol (lung-MAP)-a biomarker-driven protocol for accelerating development of therapies for squamous cell lung cancer: SWOG S1400. Clin. Cancer Res. 2015;21:1514-1524.

6. Rodon J, et al. Genomicc and transcriptomic profiling expands precision cancer medicine: the WINTHER trial. Nat. Med. 2019;25:751-758.

7. O’Shaughnessy J, et al. Phase III study of iniparib plus gemcitabine and carboplatin versus gemcitabine and carboplatin in patients with metastatic triple-negative breast cancer. J. Clin. Oncol. 2014;32:3840-3847.

8. Fröhlich, B. and Plate, J. 2000. The ccubic mouse: a new device for three-dimensional input. In Proceedings of the SIGCHI Conference on Human Factors in Computing Systems

9. Stott JRR. Orientation and disogrientation in aviation. Extreme Physiology \& Medicine. 2013; 2: 2.doi: 10.1186/2046-7648-2-2?optIn=true.

10. Newman RL, Rupert AH. The maggnitude of the spatial disorientation problem in transport airplanes. Aerosp Med Hum Perf. 2020; 91(2): 65-70.doi: 10.3357/AMHP.5442.2020.

11. Gillingham KK. A primer of vestibular function, spatial disorientation, and motion sickness. Aeromedical Reviews. 1966; 4: 1-80.

12. Lawson B, McGrath B, Rupert A, Thompson LI, Brill JC, Kell AM. A countermeasure for loss of situation awareness: Transitioning from the laboratory to the aircraft. 2016 IEEE Aerospace Conference, Big Sky, USA, 2016 
13. Rokita JL, Rathi KS, Cardenas MF, et al. Genomic Profiling of Childhood Tumor Patient-Derived Xenograft Models to Enable Rational Clinical Trial Design. Cell Rep. 2019;29(6):1675-1689.e9. doi:10.1016/j.celrep.2019.09.071

14. Shiff NJ, Oen K, Rabbani R, Lix LM. Validation of administrative case ascertainment algorithms for chronic childhood arthritis in Manitoba, Canada. Rheumatol Int. 2017;37(9):1575-1584. doi:10.1007/s00296-017-3734-1

15. Raman, Swetha and Buongervino, Samantha N. and Lane, Maria and Zhelev, Doncho V. and Zhu, Zhongyu and Cui, Hong and Martinez, Benjamin and Martinez, Daniel and Wang, Yanping and Upton, Kristen A. and Patel, Khushbu and Rathi, Komal S. and Navia, Carmen T. and Harmon, Daniel B. and Pawel, Bruce and Dimitrov, Dimiter and Maris, John M. and Julien, Jean-Philippe and Bosse, Kristopher, The D3-GPC2-PBD ADC is Potently Efficacious Against Neuroblastoma 8 and SCLC Via Engagement of a Conformational GPC2 Epitope (September 2020). Available at SSRN: https://ssrn.com/abstract=3673604 or http://dx.doi.org/10.2139/ssrn.3673604

16. Stuart S, Hickey A, Vitorio R, et al. EEye-tracker algorithms to detect saccades during static and dynamic tasks: a structured review. Physiol Meas. 2019;40(2):02TR01. Published 2019 Feb 26. doi:10.1088/1361-6579/ab02ab

17. Buxton, E. K., Vohra, S., Guo, Y., Fogleman, A., \& Patel, R. (2019). Pediatric population health analysis of southern and central Illinois region: A cross sectional retrospective study using association rule mining and multiple logistic regression. Computer methods and programs in biomedicine, 178, 145153.

18. Campbell E. Random Compiler for Fast Hamiltonian Simulation. Phys Rev Lett. 2019;123(7):070503. doi:10.1103/PhysRevLett.123.070503

19. Devkota S, Aschwanden P, Kunen A, Legendre M, Isaacs KE. CcNav: Understanding Compiler Optimizations in Binary Code. IEEE Trans Vis Comput Graph. 2021;27(2):667-677. doi:10.1109/TVCG.2020.3030357

20. Yang X, He H. An advanced compiler designed for a VLIW DSP for sensors-based systems. Sensors (Basel). 2012;12(4):4466-4478. doi:10.3390/s120404466

21. Minkovich K, Srinivasa N, Cruz-Albrecht JM, Cho Y, Nogin A. Programming time-multiplexed reconfigurable hardware using a scalable neuromorphic compiler. IEEE Trans Neural Netw Learn Syst. 2012;23(6):889-901. doi:10.1109/TNNLS.2012.2191795

22. Patel, Rushabh. "Predicting Invasive Ductal Carcinoma Using a Reinforcement Sample Learning Strategy Using Deep Learning." ArXiv:2105.12564 [Cs, Eess], May 2021. arXiv.org, http://arxiv.org/abs/2105.12564.

23. Patel, Rushabh, and Yanhui Guo. "Graph Based Link Prediction between Human Phenotypes and Genes." ArXiv:2105.11989 [Cs], May 2021. arXiv.org, http://arxiv.org/abs/2105.11989.

24. Lunter G. HMMoC-a compiler for hidden Markov models. Bioinformatics. 2007;23(18):2485-2487. doi:10.1093/bioinformatics/btm350 